\title{
SYMMETRIC DECREASING REARRANGEMENT CAN BE DISCONTINUOUS
}

\author{
FREDERICK J. ALMGREN, JR. AND ELLIOTT H. LIEB
}

Suppose $f\left(x^{1}, x^{2}\right) \geq 0$ is a continuously differentiable function supported in the unit disk in the plane. Its symmetric decreasing rearrangement is the rotationally invariant function $f^{*}\left(x^{1}, x^{2}\right)$ whose level sets are circles enclosing the same area as the level sets of $f$. Such rearrangement preserves $\mathbf{L}^{p}$ norms but decreases convex gradient integrals, e.g. $\left\|\nabla f^{*}\right\|_{p} \leq\|\nabla f\|_{p}(1 \leq p<\infty)$. Now suppose that $f_{j}\left(x^{1}, x^{2}\right) \geq 0$ $(j=1,2,3, \ldots)$ is a sequence of infinitely differentiable functions also supported in the unit disk which converge uniformly together with first derivatives to $f$. The symmetrized functions also converge uniformly. The real question is about convergence of the derivatives of the symmetrized functions. We announce that the derivatives of the symmetrized functions need not converge strongly, e.g. it can happen that $\left\|\nabla f_{j}^{*}-\nabla f^{*}\right\|_{p} \nrightarrow 0$ for every $p$. We further characterize exactly those $f$ 's for which convergence is assured and for which it can fail.

The rearrangement map $\mathscr{R}: f \rightarrow f^{*}$ in general dimensions also decreases gradient norms. For this reason alone, rearrangement has long been a basic tool in the calculus of variations and in the theory of those PDE's that arise as Euler-Lagrange equations of variational problems; it permits one to concentrate attention on radial, monotone functions and thereby reduces many problems to simple one dimensional ones. Some examples are (i) the lowest eigenfunction of the Laplacian in a ball is symmetric decreasing; (ii) the body with smallest capacity for a given volume is a ball [PS]; (iii) the optimal functions for the Sobolev and Hardy-Littlewood-Sobolev inequalities are symmetric decreasing and can be explicitly calculated [LE]. Other examples are given in [KB].

Obviously $\mathscr{R}$ is highly nonlocal, nonlinear, and nonintuitive, but the property of decreasing gradient norms would lead one to surmise that $\mathscr{R}$ is a smoothing operator in some sense. Thus when W. Ni and L. Nirenberg asked, some years ago, whether $\mathscr{R}$ is continuous in the $\mathbf{W}^{1, p}$ topology the answer appeared to be that it should be so (it is easy to prove that $\mathscr{R}$ is always a contraction in $\left.\mathbf{L}^{p}\right)$. Indeed, by an elegant analysis Coron [CJ] proved this in $\mathbf{R}^{1}$. An affirmative answer to this question would have meant that the mountain-pass lemma could be used to establish spherically symmetric solutions of certain PDE's, and Coron's result led to just such an application [RS]. Our result is that $\mathscr{R}$ is not continuous in $\mathbf{W}^{1, p}\left(\mathbf{R}^{n}\right)$ for $n \geq 2$ and it is surprising, to us at least. Since almost all applications

Received by the editors October 17, 1988 and, in revised form, November 29, 1988.

1980 Mathematics Subject Classification (1985 Revision). Primary 46E35; Secondary 26B99, 47B38. 
of $\mathscr{R}$-apart from the mountain-pass application-do not rely on continuity, our result does not have much immediate impact on applications. It reveals, however, an unexpected subtlety about the geometry of level sets of functions and shows that intuition can be very wrong. More precisely, our analysis has led us to isolate a property of $\mathrm{W}^{1, p}$ functions on their critical sets which we call co-area regularity, in terms of which we prove [AL].

MAIN TheOREM. The rearrangement map $\mathscr{R}$ is $\mathbf{W}^{1, p}\left(\mathbf{R}^{n}\right)$ continuous at a function $f$ if and only if $f$ is co-area regular.

Each $\mathbf{W}^{1, p}$ function on the line is automatically co-area regular. In higher dimensions both the regular and irregular functions are dense in $\mathbf{W}^{1, p}$.

The symmetric decreasing rearrangment of a vector valued function $f$ is defined by setting $f^{*}=|f|^{*}$. One can also replace $\mathbf{W}^{1, p}$ norms by gradient integrals of other convex integrands $\psi: \mathbf{R}^{+} \rightarrow \mathbf{R}^{+}$, i.e. $\|\nabla f\|_{p}^{p}=$ $\int|\nabla f|^{p} d \mathscr{L}^{n}\left(\mathscr{L}^{n}\right.$ is Lebesgue measure) is replaced by $\int \psi(|\nabla f|) d \mathscr{L}^{n}$. Our conclusions about continuity remain the same. However, for each $0<\alpha<1$, each $p \geq 1$, and each $n \geq 1$ we show that the rearrangement map $\mathscr{R}$ is continuous everywhere on the fractional Sobolev space $\mathbf{W}^{\alpha, p}\left(\mathbf{R}^{n}\right)$. We thus have the curious fact that co-area regularity plays a role for $\mathbf{W}^{\alpha, p}$ only when $\alpha=1$.

Definition. Suppose $f: \mathbf{R}^{n} \rightarrow \mathbf{R}^{+}$and set

$$
\mathscr{G}_{f}(y)=\int \chi_{\{f>y\}} \chi_{\{\nabla f=0\}} d \mathscr{L}^{n}
$$

for each positive number $y$; here $\chi_{A}$ denotes the characteristic function of the set $A$. Since $\mathscr{G}_{f}: \mathbf{R}^{+} \rightarrow \mathbf{R}^{+}$is nonincreasing, its distribution first derivative $\mathscr{G}_{f}^{\prime}$ is a (negative) measure. Our function $f$ is called co-area regular if and only if the measure $\mathscr{G}_{f}^{\prime}$ is purely singular with respect to $\mathscr{L}^{1}$. Otherwise $f$ is called co-area irregular.

The term co-area regular was suggested by H. Federer's "co-area formula" for the absolutely continuous function

$$
y \rightarrow \int \chi_{\{f>y\}} \chi_{\{\nabla f \neq 0\}} d \mathscr{L}^{n}
$$

which is complementary to our $\mathscr{G}_{f}$.

We also announce

THEOREM. For each $n \geq 2$ and each $0<\lambda<1$, there is (by construction) a positive constant $C$ and $a$ function $f: \mathbf{R}^{n} \rightarrow[0,1]$ in $\mathbf{C}^{n-1, \lambda}$ whose support is the unit cube $\mathbf{Q}$ such that $\mathscr{G}_{f}(y)=C(1-y)$ for each $0<y \leq 1$. In particular, the measure $\mathscr{G}_{f}^{\prime}$ is absolutely continuous with respect to $\mathscr{L}^{1}$; thus $f$ is a co-area irregular function.

Each $f$ in $\mathbf{C}^{n-1,1}$ turns out to be co-area regular and both the regular and the irregular functions are dense in $\mathrm{W}^{1, p}$ for $n \geq 2$.

The idea behind the construction above is to decompose $\mathbf{Q}$ into $2^{n}$ cubes $\mathbf{Q}_{j}$ of half the size, then decompose each of those into $2^{n} \mathbf{Q}_{j k}$ 's and so 
on. We first set $f(x)=\sum_{i=1}^{\infty} a_{i}(x) 2^{-n i}$ where $a_{i}(x)$ equals $(l-1)$ when $x$ belongs to the cube $Q_{\ldots l \ldots}$ and $l \in\left\{1, \ldots, 2^{n}\right\}$ is the index in the $i$ th position. This $f$ is not continuous but its range is uniformly spread over $(0,1)$. The second step is to "smooth" this $f$ in such a way that it belongs to $\mathrm{C}^{n-1, \lambda}$ and $\mathscr{L}^{n}\{x: \nabla f=0\}>0$.

A fuller statement of failure of continuity is the following.

THEOREM (DiscontinUity AT CO-AREA IRREGULAR FUNCTIONS). Suppose $n \geq 2$ and $f$ is a co-area irregular function belonging to $\mathbf{W}^{1, p}\left(\mathbf{R}^{n}\right)$. Then there is a sequence $f_{1}, f_{2}, f_{2}, \ldots$ of infinitely differentiable functions in $\mathbf{W}^{1, p}\left(\mathbf{R}^{n}\right)$ such that $f_{j} \rightarrow f$ in $\mathbf{W}^{1, p}\left(\mathbf{R}^{n}\right)$ as $j \rightarrow \infty$ but $f_{j}^{*} \nrightarrow f^{*}$ in $\mathbf{W}^{1, p}\left(\mathbf{R}^{n}\right)$.

The basic idea behind the proof is the following. Let $U_{j}$ be a suitable smooth approximation of $\chi\{\nabla f=0\}$ and set

$$
f_{j}(x)=f(x)+\frac{1}{2 j} U_{j}(x) \sin (j f(x))
$$

for each $x$. We confirm that $f_{j} \rightarrow f$ in $\mathbf{W}^{1, p}$ as $j \rightarrow \infty$. Defining sets $K_{(j)}(y)=\left\{x: f_{(j)}(x)>y\right\}$ for each $y$, we check for integers $m$ that $K(y)=$ $K_{j}(y)$ when $y=(2 m)(\pi / j)$ while $K(y)$ is generally a proper subset of $K_{j}(y)$ when $0<\sigma<1$ and $y=(2 m+\sigma)(\pi / j)$. Since $K_{j}$ and $K$ define $f_{j}^{*}$ and $f^{*}$ one can estimate (by using the Schwarz inequality several times and a simple Sobolev inequality) that $\left\|\nabla f_{j}^{*}-\nabla f^{*}\right\|_{p} \geq$ (constant) $\int h^{1 / 2} d \mathscr{L}^{1}$, where $\mathscr{L}^{1} \wedge h$ denotes the absolutely continuous part of $-\mathscr{G}_{f}^{\prime}$.

Now, suppose that $f_{j} \rightarrow f$ in $\mathbf{W}^{1, p}$ and that $f$ is co-area regular. As a further part of our Main Theorem we will indicate why $f_{j}^{*} \rightarrow f^{*}$ in $\mathbf{W}^{1, p}$. We infer, using Federer's co-area formula and dominated convergence, that

$$
\omega_{f}(y)=\int_{f^{-1}\{y\}} \frac{1}{|\nabla f|} d \mathscr{H}^{n-1}
$$

is well defined and finite for $\mathscr{L}^{1}$ almost every $y\left(\mathscr{C}^{n-1}\right.$ denotes Hausdorff measure) and that

$$
\int \omega_{f} d \mathscr{L}^{1}=\int \chi_{\{\nabla f \neq 0\}} d \mathscr{L}^{n}
$$

The co-area formula fails to give information about the set $\{x: \nabla f=0\}$. This missing information is contained in $\mathscr{G}_{f}$.

To compute $f^{*}$ we must compute $\sigma_{f}(y)=\int \chi_{\{f>y\}} d \mathscr{L}^{n}$ and we have $\sigma_{f}^{\prime}(y)=\mathscr{L}^{1} \wedge \omega_{f}(y)+\mathscr{G}_{f}^{\prime}(y)$. We show that $\liminf _{j \rightarrow \infty} \omega_{f_{j}}(y) \geq \omega_{f}(y)$ for almost every $y$. Let $\mathscr{L}^{1} \wedge \delta_{(j)}(y)$ be the absolutely continuous part of $\sigma_{f_{(j)}^{\prime}}^{\prime}(y)$. Since $\mathscr{G}_{f}^{\prime}$ is purely singular (this is where co-area regularity is used) we infer that

$$
\liminf _{j \rightarrow \infty} \delta_{j}(y) \geq \delta(y)
$$

To prove the convergence of $\nabla f_{j}^{*}$ to $\nabla f^{*}$ we prove the convergence of arc length of the one dimensional graphs representing these functions in polar coordinates (this is the geometrically invariant notion). It turns out (using 
several involved convexity arguments) that if we use the $\mathbf{L}^{p}$ convergence of $f_{j}^{*}$ to $f^{*}$ (so that the graphs converge pointwise) only the absolutely continuous pieces $\delta_{(j)}$ are needed and that (1) suffices for our purposes.

\section{REFERENCES}

[AL] F. Almgren and E. Lieb, Symmetric decreasing rearrangement is sometimes continuous (submitted).

[CJ] J-M. Coron, The continuity of the rearrangement in $\mathbf{W}^{1, p}(\mathbf{R})$, Ann. Scuola Norm. Sup. Pisa Sér 411 (1984), 57-85.

[KB] B. Kawohl, Rearrangements and convexity of level sets in PDE, Lecture Notes in Math., vol. 1150, Springer-Verlag, Berlin and New York, 1985, 134 pp.

[LE] E. Lieb, Sharp constants in the Hardy-Littlewood-Sobolev and related inequalities, Ann. of Math. (2) 118 (1983), 349-374.

[PS] G. Pólya and G. Szegö, Isoperimetric inequalities in mathematical physics, Ann. of Math. Studies no. 27, Princeton Univ. Press, Princeton, N. J., 1952.

[RS] B. Ruf and S. Solimini, On a class of superlinear Sturm-Liouville problems with arbitrarily many solutions, SIAM J. Math. Anal. 17 (1986), 761-771.

Department of Mathematics, Princeton University, Princeton, New Jersey 08544 